\title{
ESTIMATIVA DA PRESSÃO ARTERIAL DE CRIANÇAS DE 1 A 6 ANOS DE IDADE, COM ESPECIAL REFERÊNCIA A LARGURA DO MANGUITO
}

Dyrce Maria Rocha Martins*

MARTINS, D.M.R. Estimativa da pressão arterial de crianças de 1 a 6 anos de idade, com especial referência à largura do manguito. Rev. Esc. Enf. USP, São Paulo, 22 (3): 353-370, dez. 1988.

A autora apresenta as médias e medidas de dispersão - desvios padrão e percentis - das pressóes sistólica e diastólica de crianças de 1 a 6 anos de idade. Utilizando um critério segundo o qual a largura do manguito deve ser igual ao diàmetro do braço mais $20 \%$, aproximadamente, a autora demonstra como a largura do manguito interfere significativamente na leitura da pressäo arterial.

UNITERMOS: Pressão Arterial. Crianças.

\section{INTRODUÇÃO}

Sendo a pressão arterial (PA) um dos sinais relevantes na avaliação do estado de saúde do indivíduo, em qualquer idade, toma-se essencial a existência de padrões de referência para cada faixa etária, a fím de que os dados obtidos na prática possam ser avaliados e interpretados.

A importância da medida da PA no atendimento de crianças tem sido enfatizada por vários autores $7,8,9,13,15$, uma vez que tanto a hipertensão primária quanto a secundária podem ser detectadas na infância.

Nota-se na prática, que no atendimento à criança, a verificação da PA nem sempre é efetuada e quando incluída no exame físico, os cuidados e recomendaçóes relativas ao procedimento não são levadas em consideração ${ }^{18,22}$. Erros na determinação da PA sistólica e diastólica em crianças, como em adultos, podem advir do equipamento, da técnica, bem como das condições do cliente e da pessoa que a mede $6,9,14,18,19,25,27$.

$A$ análise da literatura pertinente ${ }^{23}$ permitiu constatar serem poucos os autores que apresentam valores da PA para crianças nas várias faixas etárias, existindo ainda, discordância entre as tabelas por eles apresentadas.

Constatou-se, também, a inexistência de estudos brasileiros sobre o assunto, e os dados sobre PA de crianças nos livros de tex to são transcritos de au tores estrangeiros ${ }^{10,11,26}$.

* Enfermeira. Mestre em Enfermagem. Professor Assistente - aposentado do Departamento de Enfergem Materno Infantil e Psiquiátrica da Escola de Enfermagem da USP - Disciplina Enfermagem Pediátrica. 
Estas constataçð̄es motivaram a realização de um primeiro estudo de PA em crianças de 0 a 1 ano de idade ${ }^{23}$. Considerando a importância de haver padrões de referência atualizados e representativos para a nossa população, decidiu-se dar continuidade a esse primeiro estudo, pesquisando a PA de crianças de 1 ano até 6 anos de idade.

Vários autores ${ }^{1,2,3,14,17,18,24,27,29}$, verificaram e confirmaram que, ao se determinar a PA sistólica e diastólica em crianças, a largura do manguito é uma variável importante a ser controlada. Por essa razão, o estudo desta variável foi controlado cuidadosamente. tivos:

Diante destas considerações foram estabelecidos para este estudo, os seguintes obje-

- determinação das médias e medidas de dispersão - desvios padrão e percentis - das pressões sistólica e diastólica de crianças de ambos os sexos, nas diferentes faixas etárias, compreendidas entre 1 a 6 anos de idade, com manguitos de largura adequada à circunferência do braço;

- verificação da influência da largura do manguito sobre os valores de PA de crianças de ambos os sexos, nas diferentes faixas etárias, compreendidas entre 1 a 6 anos de idade.

\section{METODOLOGIA}

\section{População e local}

A população foi constituída de crianças de 1 a 6 anos de idade que atendiam aos critérios de seleção descritos em 2.2.1, e frequentavam um dos locais escolhidos para a realização da pesquisa. Como campo para a realização desta, foram selecionadas três creches (duas mantidas e uma em convênio com a Prefeitura do Município de São Paulo), além de uma Escola de Educação Infantil desta Prefeitura e um Posto de Saúde da Secretaria de Saúde do Estado de São Paulo, que autorizaram a realização do estudo. Não foram levadas em consideração as condiçøes sócio-econômicas da população, e sim seu índice de crescimento, seu estado de saúde, bem como condições fisiológicas normais apresentadas no dia da coleta de dados.

O tamanho da amostra foi determinado por estaticista que, tendo como referência os valores apresentados por HAGGERTY et al. ${ }^{15}$, encontrou curva operacional para 0 teste " $t$ " com uma amostra de 45 crianças de cada sexo para cada faixa etária.

\section{Método}

\subsection{Determinação do indice de crescimento}

Para determinar o índice de crescimento utilizou-se o índice de McLaren. Este índice $e$ o indicador de crescimento resultante de pontos atribuídos à adequação percentual dos valores de peso, altura, perímetros'cefálico e braquial, quando comparados aos valores padrão. Na determinação do indice de crescimento das crianças examinadas, foram empregadas para medidas de peso, altura, perímetros cefálico e braquial, técnicas já padronizadas ${ }^{16}$. Para a comparação dos dados obtidos recorreu-se as tabelaș brasileiras de 
peso, altura e perímetro cefálico ${ }^{20} \mathrm{e}$, para o perímetro braquial, tabela estrangeira ${ }^{16}$. A classificação adotada neste estudo foi a seguinte: a soma de pontos igual a 0,1 e 2 foi considerada crescimento normal; a de 3,4 e 5, crescimento levemente prejudicado, e a de 6,7 e 8 , crescimento moderadamente prejudicado. Foram, pois, incluídas neste estudo as crianças que apresentavam índice de crescimento de 0 a 8 pontos (anexo 1).

\subsection{Verificação da pressão arterial}

Como no primeiro estudo feito por nós, o método escolhido foi o auscultatório; esta escolha deveu-se ao fato de ser de uso corrente em nosso meio, além de ser um dos métodos indiretos mais seguros para uso no campo clínico; vários autores ${ }^{2,3,4,6,19}$ já constataram serem pequenas as diferenças entre os valores da PA obtidos pelos métodos direto $e$ indireto, desde que se elimine $o$ erro que pode advir do uso de manguitos inadequados.

\subsubsection{Material}

$\mathrm{Na}$ realização desta pesquisa utilizou-se o seguinte material:

- um esfigmomanômetro aneróide Tycus;

- um esfigmomanômetro de mercúrio;

- um estetoscópio infantil com diafragma medindo $3,5 \mathrm{~cm}$ de diâmetro;

- um conjunto de onze manguitos, cujas bolsas de borracha variavam de $0,5 \mathrm{em}$ $0,5 \mathrm{~cm}$ de largura e observadas as seguintes proporçð̄es: $4,5 \mathrm{X} 12,0 ; 5,0 \mathrm{X} 10,5 ; 5,5 \mathrm{X}$ X 13,$0 ; 6,0 \times 12,5 ; 6,5 \times 13,5 ; 7,0 \times 16,0 ; 7,5 \times 15,5 ; 8,0 \times 15,6 ; 8,5 \times 18,5 ; 9,0 \times$ X 19,$0 ; 9,5 \times 20,0$;

- trena de fibra de vidro;

- toeza;

- balança;

- termômetro clínico;

- ficha de registro de dados (anexo 2).

Para controlar as variáveis mais relevantes que podem interferir na exatidão da leitura das pressões sistólica e diastólica em crianças, foram levados em consideração os seguintes critérios e condiçð̃es:

\section{Condição dos instrumentos:}

O manômetro aneróide utilizado na pesquisa foi testado contra o aparelho de mercúrio antes do início da coleta de dados, e aferido após ser utilizado em cada grupo de 30 crianças aproximadamente, durante toda a coleta de dados. Manguitos de dimensões apropriadas, isto é, de comprimento suficiente para envolver o braço da criança e de largura adequada para cobrir a extensão proporcional ao diâmetro do braço mais o acréscimo medio de $20 \%$. Neste estudo a idade não foi o ponto de referência para a seleção do manguito, mas sim as dimensões do braço de cada criança. Com a finalidade de testar a influência da largura do manguito sobre os valores das pressões sistólica e diastólica, foram utilizados, além do manguito adequado, outros dois manguitos considerados inadequados, um, cerca de um centímetro mais estreito e outro, cerca de um centímetro mais largo que o adequado, o que explica a variação das medidas dos 11 manguitos acima referidos. 
No anexo é apresentada uma relação de perímetros braquiais e larguras de manguitos adequados correspondentes, segundo o critério do acréscimo médio de $20 \%$ sobre o diâmetro do braço.

\section{Critério de seleção das crianças}

As crianças incluídas neste estudo foram aquelas que:

- frequentavam um dos locais escolhidos;

- apresentavam índice de crescimento de 0 a 8 pontos;

- não se encontravam sob cuidados médicos devido a distúrbios de saúde;

- no dia da coleta de dados não apresentavam alterações fisiológicas card íacas, térmicas e/ou digestivas. Para verificação das condiçбes fisiologicas de cada criança, mediu-se a temperatura e controlou-se a frequência cardíaca e respiratória, e para averiguação das condições digestivas foi consultada a mãe ou pessoa responsável pela criança e/ou a própria criança. Enquanto se faziam estas verificaçóes, a examinadora procurou estabelecer relacionamento e comunicação com a criança, permitindo-lhe ainda, que repousasse pelo menos por cinco minutos, antes de proceder às leituras da PA.

\section{Condiçôes da criança}

Durante a leitura da PA a criança devia estar: acordada e sem manifestação de sono, livre do desconforto de roupas sujas ou molhadas, no intervalo das refeiçôes, quieta e apresentando relaxamento muscular do membro escolhido para a tomada da PA, com fisionomia tranquiila ${ }^{22}$. A posição da criança podia ser decúbito dorsal ou sentada, com 0 . braço apoiado ao nível do coração.

\section{Condições para a leitura da $P A$}

Neste estudo foram observadas as recomendaçoes aprovadas pela American Heart Association em 1967, não somente no referente a calibragem do aparelho, posição do esfigmomanometro em relação ao examinador, seleção e aplicação do manguito e do estetoscópio no braço da criança, velocidade ao insuflar e ao esvaziar a bolsa de borracha do manguito, como também todas as recomendações relativas à sequência a ser observada durante a leitura das pressōes sistólica e diastólica ${ }^{18}$. Assim, determinou-se em primeiro lugar a pressão sistólica pelo método palpatório, e a seguir foi insuflado o manguito, aproximadamente $30 \mathrm{mmHg}$ acima daquele valor, procedendo-se então, três vezes à leitura das pressð̃es sistólica e diastólica pelo método auscultatório. Utilizou-se primeiro o manguito considerado adequado; em seguida, repetindo-se os mesmos procedimentos, empregou-se um manguito cerca de um centímetro mais estreito que o adequado e por último, um manguito cerca de um centímetro mais largo que o adequado.

Todas as leituras foram feitas pela mesma examinadora, assessorada por uma assistente ou pela mãe da criança, que pocurou mantê-la em condição de ser examinada, isto é, quieta e tranquila.

Com objetivo de serem conseguidos tranquilidade e relaxamento muscular da criança, a examinadora e a assistente ou mãe, utilizaram contacto físico, comunicação, brinquedos, gestos, bem como o próprio material de exame, a fim de que a criança se acostumasse à examinadora, se familiarizasse com os instrumentos do exame e se manti- 
vesse calma diante da situação apresentada pelo aparelho em funcionamento em seu braço no momento das leituras e dessa forma colaborasse.

Critério para interpretaçăo dos valores da $P A$.

Foi o mesmo das recomendaçס̄es aprovadas pela American Heart Association em 1967, a saber: o ponto onde se ouviram os primeiros sons de Korotkoff, ao ser esvaziado o manguito, foi considerado como a pressão sistólica e o ponto onde os sons mudaram de intensidade, "abafamento", foi considerado como pressão diastólica. Quando a pressão sistólica por palpação resultou mais alta que a percebida pela ausculta, a primeira foi considerada como a pressão sistólica ${ }^{18}$.

\subsection{Tratamento dos dados}

A análise estatística dos dados visou a determinação média, desvio padrão e diferentes percentis para as pressőes sistólica e diastólica de meninos e meninas em cada faixa etária. Foram estabelecidos contrastes entre as médias e analisada a diferença entre duas medias, através do teste " $t$ " para observaçð̃es pareadas dependentes. 0 núvel de significância estabelecido para estes testes foi de $1 \%^{5,28}$.

\section{RESULTADOS E COMENTÁRIOS}

\subsection{Caracteristica da populaçâo}

Os resultados apresentados neste estudo baseiam-se em 5.382 leituras da PA, em 598 crianças de 1 a 6 anos de idade. Das crianças examinadas, 292 eram do sexo masculino e 306 do sexo feminino.

Como o indice de estudo foi utilizado para excluir as crianças com crescimento muito prejudicado, a população estudada incluiu $52,01 \%$ de crianças com crescimento normal, 37,96\% com crescimento levemente prejudicado e $10,10 \%$ com crescimento moderadamente prejudicado.

3.2. Valores médios e medidas de dispersão da PA de crianças de 12 a 84 meses de idade.

Como é sabido, o critério de referir um determinado índice, considerando apenas a media aritmética é de duvidosa utilidade, uma vez que somente uma pequena porcentagem de indivíduos apresenta medidas iguais à média. Para minimizar estes problemas, são usadas medidas de dispersão. Estas estabelecem diferentes valores normais de variação para determinada medida. O conhecimento das medidas de dispersão para valores que controlam variáveis de saúde é de grande importância na prática diária, sendo que as medidas de dispersão mais usadas para valores da pressão arterial são: desvio padrão e percentil.

O desvio padrão é a medida quadrática entre os afastamentos encontrados com relação a média aritmética. Em uma distribuição normal, o desvio padrão, tomado com duplo sinal \pm em tomo da média aritmética, abrange $68,27 \%$ das probabilidades de fre- 
quiência, se forem utilizados duas vezes o desvio padrão com duplo sinal \pm , se rão abrangidas $95,45 \%$ da probabilidade de ocorrência.

O percentil indica a posição que uma determinada medida ocupa numa série de 100 . Por exemplo, o $90^{\circ}$ percentil para a PA de uma criança indica que há 90 crianças com PA mais baixa e 10 com PA mais alta do que a dela. $O$ percentil 50 representa o ponto médio, isto é, indica que há 50 indivíduos com valores abaixo e $50 \mathrm{com}$ valores acima do $\operatorname{seu}^{28}$.

Qualquer uma destas medidas pode ser adotada, desde que se tenham padrós de referência que permitam interpretar os dados obtidos na prática.

Para atender aos objetivos deste estudo foram determinadas em primeiro lugar, as medias e os desvios padrão para as pressoes sistólica e diastólica de meninos e meninas das diferentes faixas etárias, sendo utilizados para esse fim, manguitos de dimensర̄es adequadas.

Apesar da análise estatística ter demonstrado não existir diferença significativa entre as médias das pressరoes sistólica e diastólica de meninos e meninas, na Tabela 1 são apresentadas as médias e os desvios padrão destas medidas, segundo o sexo em cada faixa etária.

TABELA 1

MÉDIAS E DESVIOS PADRÃO* DA PA DE CRIANÇAS DE 12 - 84 MESES DE IDADE.

\begin{tabular}{|c|c|c|c|c|}
\hline \multirow{2}{*}{$\begin{array}{l}\text { Idade } \\
\text { (meses) }\end{array}$} & \multicolumn{2}{|c|}{ Pressão Sistólica (mmHg) } & \multicolumn{2}{|c|}{ Pressão Diastólica (mmHg) } \\
\hline & Meninos & Meninas & Meninos & Meninas \\
\hline $\begin{array}{l}12 \vdash 24 \\
24 \vdash 36 \\
36 \vdash 48 \\
48 \vdash 60 \\
60 \vdash 72 \\
72 \vdash 84\end{array}$ & $\begin{array}{l}107,7(6,8) \\
107,2(7,5) \\
108,2(7,3) \\
110,4(7,6) \\
108,1(8,8) \\
109,4(8,7)\end{array}$ & $\begin{array}{l}104,8(9,0) \\
105,5(6,9) \\
107,6(7,1) \\
106,0(7,4) \\
107,9(8,4) \\
107,1(8,4)\end{array}$ & $\begin{array}{l}75,3(6,9) \\
75,6(6,1) \\
77,7(7,5) \\
79,5(6,2) \\
77,6(6,1) \\
79,1(7,2)\end{array}$ & $\begin{array}{l}75,8(5,8) \\
75,3(5,9) \\
77,7(6,0) \\
76,2(6,0) \\
78,1(6,6) \\
78,0(6,0)\end{array}$ \\
\hline
\end{tabular}

* Os desvios padrão são apresentados entre parêntesis.

As medias obtidas para as diferentes faixas etárias, tanto de meninos como de meninas, apresentam pequenas oscilaçðes entre si, estatísticamente ño significativas.

Estes resultados vieram confirmar que a PA de criança é pouco mais baixa do que a do adulto. Estes resultados são diferentes aos apresentados em tabelas estrangeiras, principalmente com respeito aos valores das pressðes sistólica e diastólica deste estudo, que não apresentam crescimento homogêneo, como se observa nos dados de outros autores $^{1,12,15}$.

Além do desvio padrão, foram calculados os diferentes percentis para os valores das pressðes sistólica e diastólica de meninos e meninas nas diferentes faixas etárias. $O$ percentil é a medida mais utilizada intemacionalmente, para representar valores da $\mathrm{PA}^{7}$. Nas ta- 
belas $2,3,4$ e 5 são apresentados os diferentes valores dos percentis das pressões sistólica e diastólica de meninos e meninas de $12-84$ meses de idade.

TABELA 2

DIFERENTES PERCENTIS PARA A PRESSÃO SISTOLICA (mmHg) DE MENINOS DE 12 - 84 MESES DE IDADE.

\begin{tabular}{lrrrrrrr}
\hline \multirow{2}{*}{$\begin{array}{l}\text { Idade } \\
\text { (meses) }\end{array}$} & \multicolumn{7}{c}{ Percentil } \\
\cline { 2 - 7 } & \multicolumn{1}{c}{$\mathbf{5}$} & $\mathbf{1 0}$ & $\mathbf{2 5}$ & $\mathbf{5 0}$ & $\mathbf{7 5}$ & $\mathbf{9 0}$ & $\mathbf{9 5}$ \\
\hline $12 \vdash 24$ & 96,8 & 98,5 & 103,2 & 108,6 & 113,1 & 116,8 & 118,9 \\
$24 \vdash 36$ & 95,1 & 95,2 & 100,2 & 105,3 & 110,3 & 115,4 & 120,2 \\
$36 \vdash 48$ & 96,4 & 97,7 & 102,3 & 109,7 & 113,9 & 118,4 & 120,9 \\
$48 \vdash 60$ & 97,6 & 100,1 & 105,7 & 110,9 & 115,7 & 119,9 & 124,1 \\
$60 \vdash 72$ & 95,3 & 96,6 & 100,9 & 108,6 & 114,9 & 119,4 & 122,6 \\
$72 \vdash 84$ & 95,4 & 97,1 & 102,7 & 110,7 & 116,9 & 121,2 & 123,1 \\
\hline
\end{tabular}

TABELA 3

DIFERENTES PERCENTIS PARA A PRESSÃO DIASTÓLICA (mmHg) DE MENINOS DE 12 - 84 MESES DE IDADE.

\begin{tabular}{lccccccc}
\hline \multirow{2}{*}{$\begin{array}{l}\text { Idade } \\
\text { (meses) }\end{array}$} & \multicolumn{7}{c}{ Percentil } \\
\cline { 2 - 8 } & $\mathbf{5}$ & $\mathbf{1 0}$ & $\mathbf{2 5}$ & $\mathbf{5 0}$ & $\mathbf{7 5}$ & $\mathbf{9 0}$ & $\mathbf{9 5}$ \\
\hline $12 \vdash 24$ & 62,8 & 66,2 & 71,3 & 76,4 & 81,4 & 84,9 & 87,4 \\
$241-36$ & 65,1 & 65,2 & 70,2 & 75,2 & 75,5 & 85,0 & 85,3 \\
$36 \vdash 48$ & 68,1 & 70,4 & 73,8 & 78,4 & 83,2 & 86,9 & 88,5 \\
$48 \vdash 60$ & 69,3 & 71,5 & 76,1 & 80,7 & 84,5 & 87,7 & 88,8 \\
$60 \vdash 72$ & 66,9 & 69,9 & 73,6 & 78,4 & 82,8 & 85,9 & 89,1 \\
$72 \vdash 84$ & 66,2 & 68,1 & 75,1 & 79,8 & 85,3 & 88,1 & 89,1 \\
\hline
\end{tabular}

TABELA 4

DIFERENTES PERCENTIS PARA A PRESSÃO SISTÓLICA (mmHg) DE MENINAS DE 12 - 84 MESES DE IDADE.

\begin{tabular}{lccccccc}
\hline \multirow{2}{*}{$\begin{array}{l}\text { Idade } \\
\text { (meses) }\end{array}$} & \multicolumn{7}{c}{ Percentil } \\
\cline { 2 - 8 } & $\mathbf{5}$ & $\mathbf{1 0}$ & $\mathbf{2 5}$ & $\mathbf{5 0}$ & $\mathbf{7 5}$ & $\mathbf{9 0}$ & $\mathbf{9 5}$ \\
\hline $12 \vdash 24$ & 90,3 & 93,8 & 99,1 & 105,2 & 110,2 & 114,8 & 118,1 \\
$24 \vdash 36$ & 95,0 & 95,2 & 100,1 & 105,2 & 110,1 & 110,4 & 115,2 \\
$36 \vdash 48$ & 95,9 & 97,3 & 101,9 & 108,1 & 113,2 & 117,9 & 120,0 \\
$48 \vdash 60$ & 92,5 & 95,7 & 100,9 & 106,7 & 110,4 & 116,3 & 119,4 \\
$60 \vdash 72$ & 95,4 & 97,2 & 101,6 & 109,5 & 115,6 & 119,5 & 122,4 \\
$72 \vdash 84$ & 96,1 & 97,3 & 100,7 & 106,9 & 113,4 & 119,5 & 122,8 \\
\hline
\end{tabular}


DIFERENTES PERCENTIS PARA A PRESSÃO DIASTOLICA (mmHg) DE MENINAS DE 12 - 84 MESES DE IDADE.

\begin{tabular}{lccccccc}
\hline \multirow{2}{*}{$\begin{array}{l}\text { Idade } \\
\text { (meses) }\end{array}$} & \multicolumn{7}{c}{ Percentil } \\
\cline { 2 - 8 } & $\mathbf{5}$ & $\mathbf{1 0}$ & $\mathbf{2 5}$ & $\mathbf{5 0}$ & $\mathbf{7 5}$ & $\mathbf{9 0}$ & $\mathbf{9 5}$ \\
\hline $12 \vdash 24$ & 66,8 & 68,6 & 72,0 & 76,3 & 80,9 & 85,7 & $\mathbf{8 7 , 9}$ \\
$24 \vdash 36$ & 65,1 & 65,2 & 70,0 & 75,1 & 80,1 & 80,4 & 80,5 \\
$36 \vdash 48$ & 68,3 & 70,7 & 73,7 & 78,1 & 83,1 & 87,0 & 88,5 \\
$48 \vdash 60$ & 66,9 & 70,0 & 72,7 & 76,7 & 80,3 & 84,4 & 86,9 \\
$60 \vdash 72$ & 66,6 & 69,1 & 73,1 & 78,9 & 84,3 & 87,7 & 88,8 \\
$72 \vdash 84$ & 66,8 & 69,5 & 75,4 & 78,9 & 82,9 & 86,1 & $\mathbf{8 8 , 0}$ \\
\hline
\end{tabular}

Consideram se normais os dados da PA que se enquadram entre os percentis 5 e 95 . Valores acima do percentil 95 são considerados indicativos de hipertensão arterial ${ }^{7}$.

Os valores do $50^{\circ}$ e e $90^{\circ}$ percentis para as pressð̃es sistólica e diastólica obtidos neste estudo, são semelhantes ou apenas ligeiramente mais elevados de que os valores apresentados por autores estrangeiros. Os referidos autores só apresentam valores dos percentis da PA para crianças a partir de 2 anos de idade?

\subsection{Influência da largura do manguito sobre os valore da PA de crianças de 12 a 84 meses de idade.}

Para atender ao segundo objetivo deste estudo, em todas as crianças examinadas, as leituras da PA foram realizadas com mais dois manguitos de largura diferente daquela do manguito adequado; um manguito mais estreito, com acréscimo médio aproximado de $17,02 \%$ e outro mais largo, com acréscimo médio aproximado de $23,21 \%$, em relação ao diâmetro do braço da criança. Este fato ofereceu elementos para demonstrar como e quanto a largura do manguito pode influir nos valores das pressões sistolica e diastólica de crianças em todas as faixas etárias estudadas.

As médias e desvios padrão da PA, de meninos e meninas de 1 a 6 anos de idade, conforme largura do manguito, são apresentados nas tabelas 6 e 7 .

Como se pode observar, os valores das pressões sistólica e diastólica sofreram influência para mais ou para menos, inversamente relacionada à largura do manguito. As diferenças nos valores de PA foram analisados, segundo o teste " $t$ " para observações pareadas dependentes, observando-se significancia ao nivel de $1 \%$. As tabelas $8,9,10$ e 11 evidenciam os resultados destes testes.

Os dados das tabelas 8 e 9 permitem constatar que a in fluência da largura do manguito sobre os valores das pressð̃es sistólica e diastólica foi maior quando se usou manguito mais estreito do que quando se usou manguito mais largo que o adequado (tabelas $10 \mathrm{e}$ 11). Os valores das pressões sistólica e diastólica elevaram-se quando foi usado manguito mais estreito, como segue: pressão sistólica, amplitude de variação média de $16,1 \mathrm{mmHg}$ a $11,1 \mathrm{mmHg}$ para meninos e de $28,2 \mathrm{mmHg}$ a $15,5 \mathrm{mmHg}$, para meninas, pressão diastólica: de $11,9 \mathrm{mmHg}$ a $9,3 \mathrm{mmHg}$ para meninos e de $18,8 \mathrm{mmHg}$ a $6,7 \mathrm{mmHg}$ para as meninas. Quando se usou manguito mais largo que o adequado os valores das pressões sistólica 
MEDIAS E DESVIOS PADRÃO DA PRESSÃO ARTERIAL DE MENINOS DE 12 - 84 MESES DE IDADE, CONFORME LARGURA DO MANGUITO.

\begin{tabular}{|c|c|c|c|}
\hline \multirow{2}{*}{$\begin{array}{l}\text { Idade } \\
\text { (meses) }\end{array}$} & \multirow{2}{*}{$\begin{array}{l}\text { Largura do Manguito } \\
\text { Acréscimo médio à } \\
\text { medida do diâmetro } \\
\text { do braço (\%) }\end{array}$} & \multicolumn{2}{|c|}{ Pressão Arterial } \\
\hline & & $\begin{array}{l}\text { Sistólica } \\
\text { ( } \mathbf{m m H g} \text { ) }\end{array}$ & $\begin{array}{c}\text { Diastólica } \\
\text { (mmHg) }\end{array}$ \\
\hline $12 \vdash 24$ & $\begin{array}{l}16,93(1,0) \\
19,94(6,5)^{* *} \\
23,28(0,5)\end{array}$ & $\begin{array}{c}123,8(11.6) \\
107,7(6.8) \\
97,5(6,6)\end{array}$ & $\begin{array}{l}84,6(8,5) \\
75,3(6,9) \\
66,4(6,4)\end{array}$ \\
\hline $24 \vdash 36$ & $\begin{array}{l}16,99(0,8) \\
19,48(0,5) * * \\
23,24(0,5)\end{array}$ & $\begin{array}{c}122,9(12,2) \\
107,5(7,5) \\
97,0(7,8)\end{array}$ & $\begin{array}{l}86,7(9,3) \\
75,6(6,1) \\
68,0(6,8)\end{array}$ \\
\hline $36 \vdash 48$ & $\begin{array}{l}17,06(0,7) \\
20,19(0,5) * * \\
23,34(0,5)\end{array}$ & $\begin{array}{c}119,5(10,9) \\
108,2(7,3) \\
98,4(5,6)\end{array}$ & $\begin{array}{l}86,6(8,7) \\
77,7(7,5) \\
71,8(4,9)\end{array}$ \\
\hline $48 \vdash 60$ & $\begin{array}{l}17,01(0,5) \\
20,11(0,4) * * \\
23,19(0,5)\end{array}$ & $\begin{array}{l}123,8(14,8) \\
110,4(7,6) \\
101,0(6,5)\end{array}$ & $\begin{array}{l}91,1(10,7) \\
79,5(6.2) \\
73,2(5.7)\end{array}$ \\
\hline $60 \vdash 72$ & $\begin{array}{l}17,16(0,7) \\
20,20(0,4) * * \\
23,14(0,6)\end{array}$ & $\begin{array}{l}122,3(14,4) \\
108,1(8,8) \\
101,1(6,9)\end{array}$ & $\begin{array}{l}89,5(11,2) \\
77,6(6,1) \\
74,8(5,2)\end{array}$ \\
\hline $72 \vdash 84$ & $\begin{array}{l}17,14(0,5) \\
20,12(0,4) * * \\
23,09(0,5)\end{array}$ & $\begin{array}{l}128,5(12,5) \\
109,4(8,7) \\
101,8(7,9)\end{array}$ & $\begin{array}{l}88,5(9,9) \\
79,1(7,2) \\
74,6(5,9)\end{array}$ \\
\hline
\end{tabular}

* Os desvios padrão são apresentados entre parênteses.

** Largura adequada do manguito correspondendo ao acréscimo médio aproximado de $20 \%$ sobre o diâmetro do braço.

e diastólica das mesmas crianças diminuíram significativamente, numa amplitude de variação media de: pressão sistólica, $10,2 \mathrm{mmHg}$ a $7,0 \mathrm{mmHg}$ para meninos e de $9,8 \mathrm{mmHg}$ a $7,5 \mathrm{mmHg}$ para meninas; pressão diastólica: de $8,9 \mathrm{mmHg}$ a $2,8 \mathrm{mmHg}$ para meninos e de $8,2 \mathrm{mmHg}$ a $5,0 \mathrm{mmHg}$ para meninas.

Pode-se assim, verificar que a influência da largura do manguito é maior sobre a pressão sistólica do que sobre a diastólica. Resultados semelhantes são apresentados por autores estrangeiros ${ }^{17,27}$.

Estes resultados vêm reafirmar a necessidade enfatizada por muitos autores quanto à importância de se escolher manguito adequado à dimensão do braço da criança, ao se proceder à leitura da pressão arterial.

Por esta razão concorda-se com aqueles que afirmam ser o diâmetro do braço o fator que determina a seleção do manguito e não a idade da criança ${ }^{18,22,23,25,27,29}$. 
MÉdIAS E DESVIOS PADRÃO DA PRESSÃO ARTERIAL DE MENINAS DE 12 - 84 MESES DE IDADE, CONFORME LARGURA DO MANGUITO.

\begin{tabular}{|c|c|c|c|}
\hline \multirow{2}{*}{$\begin{array}{l}\text { Idade } \\
\text { (meses) }\end{array}$} & \multirow{2}{*}{$\begin{array}{l}\text { Largura do Manguito } \\
\text { Acréscimo médio à } \\
\text { medida do diâmetro } \\
\text { do braço }(\%)\end{array}$} & \multicolumn{2}{|c|}{ Pressão Arterial } \\
\hline & & $\begin{array}{l}\text { Sistólica } \\
(\mathbf{m m H g})\end{array}$ & $\begin{array}{c}\text { Diastólica } \\
\text { (mmHg) }\end{array}$ \\
\hline $12 \vdash 24$ & $\begin{array}{l}17,08(1,0) \\
19,98(0,6) * * \\
23,40(0,6)\end{array}$ & $\begin{array}{c}133,0(22,6) \\
104,8(9,0) \\
95,0(7,8)\end{array}$ & $\begin{array}{l}94,6(15,0) \\
75,8(5,8) \\
67,6(6,4)\end{array}$ \\
\hline $24 \vdash 36$ & $\begin{array}{l}16,82(0,8) \\
19,94(0,5)^{* *} \\
23,25(0,6)\end{array}$ & $\begin{array}{c}122,0(17,2) \\
105,6(6,9) \\
96,5(7,1)\end{array}$ & $\begin{array}{l}88,1(14,2) \\
75,3(5,9) \\
67,3(6,5)\end{array}$ \\
\hline $36 \vdash 48$ & $\begin{array}{l}16,85(1,0) \\
20,08(0,5)^{* *} \\
23,19(0,5)\end{array}$ & $\begin{array}{c}125,6(14,8) \\
107,6(7,1) \\
98,3(6,7)\end{array}$ & $\begin{array}{l}90,4(10,8) \\
77,7(6,0) \\
71,3(6,8)\end{array}$ \\
\hline $48 \vdash 60$ & $\begin{array}{l}17,10(0,5) \\
20,19(0,5)^{* *} \\
23,32(0,6)\end{array}$ & $\begin{array}{c}121,7(16,5) \\
106,0(7,4) \\
98,5(7,7)\end{array}$ & $\begin{array}{l}88,4(13,0) \\
76,2(6,0) \\
70,8(5,8)\end{array}$ \\
\hline $60 \vdash 72$ & $\begin{array}{l}17,08(0,5) \\
20,08(0,5)^{* *} \\
23,16(0,7)\end{array}$ & $\begin{array}{c}123,4(14,7) \\
107,9(8,4) \\
99,9(7,1)\end{array}$ & $\begin{array}{l}89,6(9,9) \\
78,1(6,6) \\
72,9(6,6)\end{array}$ \\
\hline $72 \vdash 84$ & $\begin{array}{l}17,04(0,4) \\
20,06(0,4)^{* *} \\
23,09(0,5)\end{array}$ & $\begin{array}{c}123,6(15,7) \\
107,1(8,4) \\
99,4(6,2)\end{array}$ & $\begin{array}{l}89,7(10,1) \\
78,0(6,0) \\
73,0(6,0)\end{array}$ \\
\hline
\end{tabular}

* Os desvios padrão são apresentados entre parênteses.

** Largura adequada do manguito, correspondendo ao acréscimo médio aproximado de $20 \%$ sobre o diâmetro do braço.

O presente estudo evidenciou, também, ser o método auscultatório, com utilização de aparelho comum, praticável em crianças, mesmo naquelas de baix a idade; a coleta de dados, permitiu confirmar que a técnica utilizada em crianças é mais complexa que a empregada em adultos, seja devido a variações do membro, mudança dos valores normais de acordo com a idade ou, frequentemente, completa falta de cooperação por parte da criança. Adequando a técnica ds características de desenvolvimento da criança, podem-se obter resultados exatos e reproduzíveis ${ }^{14}$ e 23 .

\section{CONCLUSÕES}

Os resultados obtidos no presente estudo permitem as conclusð̃es que seguem:

- Os valores médios da PA de crianças de 1 a 6 anos de idade, com índice de crescimento de 0 a 8 pontos determinados pelo método auscultatório, com manguito adequa- 
TABELA 8

RESULTADOS RELEVANTES PARA OS TESTES DE DIFERENÇA DE VALORES DAS PRESSŌES SISTÓLICA E DIASTÓLICA DE MENINOS, POR GRUPO ETÁRIO, COM MANGUTTO ADEQUADO E MAIS ESTREITO

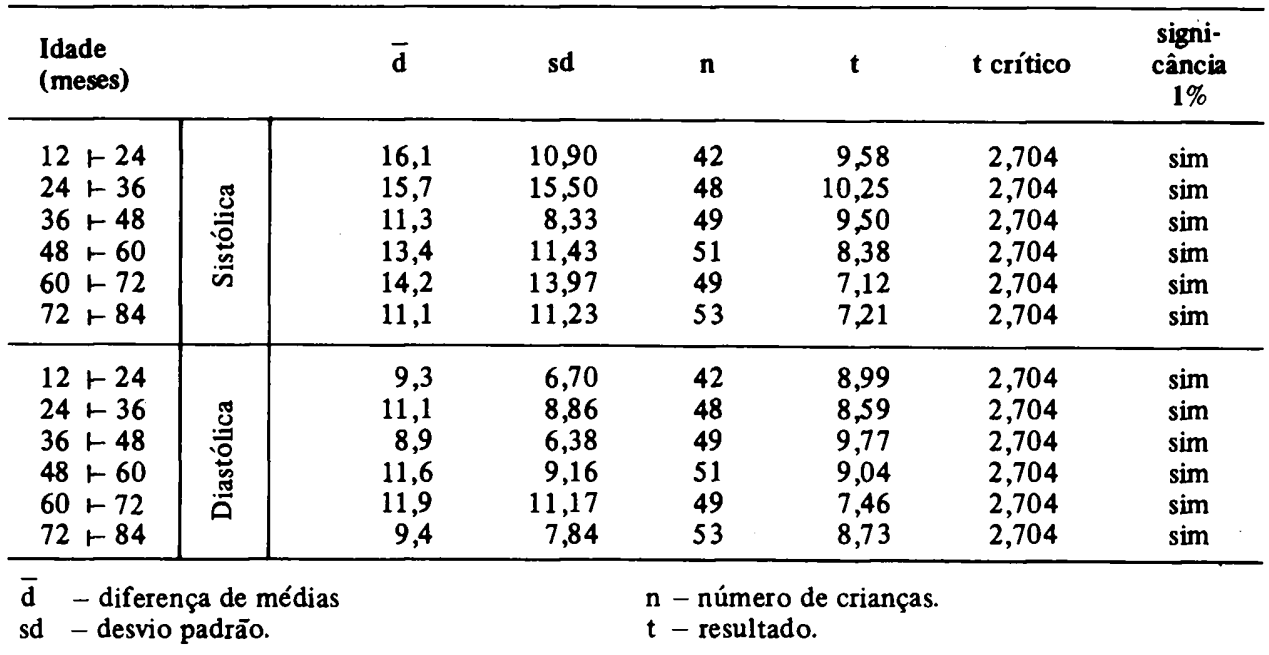

TABELA 9

RESULTADOS RELEVANTES PARA OS TESTES DE DIFERENÇA DE VALORES DAS PRESSOES SISTÓLICA E DIASTOLICA DE MENINAS, POR GRUPO ETÁRIO, COM MANGUTTO ADEQUADO E MAIS ESTREITO

\begin{tabular}{|c|c|c|c|c|c|c|c|}
\hline $\begin{array}{l}\text { Idade } \\
\text { (meses) }\end{array}$ & & $\bar{d}$ & sd & $\mathbf{n}$ & $\mathbf{t}$ & t crítico & $\begin{array}{c}\text { signi- } \\
\text { cância } \\
1 \%\end{array}$ \\
\hline $\begin{array}{l}12 \vdash 24 \\
24 \vdash 36 \\
36 \vdash 48 \\
48 \vdash 60 \\
60 \vdash 72 \\
72 \vdash 84\end{array}$ & 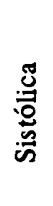 & $\begin{array}{l}28,2 \\
16,5 \\
18,0 \\
15,7 \\
15,5 \\
16,5\end{array}$ & $\begin{array}{l}19,00 \\
16,78 \\
14,36 \\
15,09 \\
11,03 \\
15,50\end{array}$ & $\begin{array}{l}43 \\
47 \\
60 \\
50 \\
51 \\
55\end{array}$ & $\begin{array}{r}9,72 \\
6,72 \\
9,71 \\
7,14 \\
10,03 \\
7,89\end{array}$ & $\begin{array}{l}2,704 \\
2,704 \\
2,704 \\
2,704 \\
2,704 \\
2,704\end{array}$ & $\begin{array}{l}\text { sim } \\
\text { sim } \\
\text { sim } \\
\text { sim } \\
\text { sim } \\
\text { sim }\end{array}$ \\
\hline $\begin{array}{l}12 \vdash 24 \\
24 \vdash 36 \\
36 \vdash 48 \\
48 \vdash 60 \\
60 \vdash 72 \\
72 \vdash 84\end{array}$ & 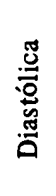 & $\begin{array}{r}18,8 \\
12,8 \\
12,7 \\
12,2 \\
11,5 \\
6,7\end{array}$ & $\begin{array}{r}13,20 \\
14,80 \\
10,90 \\
11,71 \\
8,36 \\
11,30\end{array}$ & $\begin{array}{l}43 \\
47 \\
60 \\
50 \\
51 \\
55\end{array}$ & $\begin{array}{l}9,33 \\
5,93 \\
9,03 \\
7,44 \\
9,83 \\
4,40\end{array}$ & $\begin{array}{l}2,704 \\
2,704 \\
2,704 \\
2,704 \\
2,704 \\
2,704\end{array}$ & $\begin{array}{l}\text { sim } \\
\text { sim } \\
\text { sim } \\
\text { sim } \\
\text { sim } \\
\text { sim }\end{array}$ \\
\hline $\begin{array}{l}\text { - difer } \\
\text { - desvi }\end{array}$ & & & & & 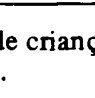 & & \\
\hline
\end{tabular}


TABELA 10

RESULTADOS RELEVANTES PARA OS TESTES DE DIFERENÇA DE VALORES DAS PRESSÓES SISTOLICA E DIASTÓLICA DE MENINOS, POR GRUPO ETÁRIO, COM MANGUITO ADEQUADO E MAIS LARGO.

\begin{tabular}{|c|c|c|c|c|c|c|c|}
\hline $\begin{array}{l}\text { Idade } \\
\text { (meses) }\end{array}$ & & $\vec{d}$ & sd & $\mathbf{n}$ & $\mathbf{t}$ & t crítico & $\begin{array}{c}\text { signi- } \\
\text { cância } \\
1 \%\end{array}$ \\
\hline $\begin{array}{l}12 \vdash 24 \\
24 \vdash 36 \\
36 \vdash 48 \\
48 \vdash 60 \\
60 \vdash 72 \\
72 \vdash 84\end{array}$ & 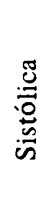 & $\begin{array}{r}10,2 \\
10,2 \\
9,8 \\
9,4 \\
7,0 \\
7,6\end{array}$ & $\begin{array}{l}5,70 \\
5,70 \\
5,49 \\
6,44 \\
6,49 \\
4,19\end{array}$ & $\begin{array}{l}42 \\
48 \\
49 \\
51 \\
49 \\
53\end{array}$ & $\begin{array}{r}11,60 \\
12,25 \\
12,50 \\
10,42 \\
7,55 \\
13,21\end{array}$ & $\begin{array}{l}2,704 \\
2,704 \\
2,704 \\
2,704 \\
2,704 \\
2,704\end{array}$ & $\begin{array}{l}\text { sim } \\
\text { sim } \\
\text { sim } \\
\text { sim } \\
\text { sim } \\
\text { sim }\end{array}$ \\
\hline $\begin{array}{l}12 \vdash 24 \\
24 \vdash 36 \\
36 \vdash 48 \\
48 \vdash 60 \\
60 \vdash 72 \\
72 \vdash 84\end{array}$ & 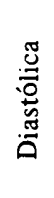 & $\begin{array}{l}8,9 \\
7,6 \\
5,9 \\
6,3 \\
2,8 \\
4,5\end{array}$ & $\begin{array}{l}6,36 \\
6,74 \\
5,15 \\
5,78 \\
5,78 \\
4,92\end{array}$ & $\begin{array}{l}42 \\
48 \\
49 \\
51 \\
49 \\
53\end{array}$ & $\begin{array}{l}9,07 \\
7,73 \\
8,02 \\
7,78 \\
3,39 \\
6,66\end{array}$ & $\begin{array}{l}2,704 \\
2,704 \\
2,704 \\
2,704 \\
2,704 \\
2,704\end{array}$ & $\begin{array}{l}\operatorname{sim} \\
\text { sim } \\
\text { sim } \\
\text { sim } \\
\text { sim } \\
\text { sim }\end{array}$ \\
\hline
\end{tabular}

TABELA 11

RESULTADOS RELEVANTES PARA OS TESTES DE DIFERENCCA DE VA.LORES DAS PRESSOES SISTÓLICA E DIASTOLICA DE MENINAS, POR GRUPO ETÁRIO, COM MANGUITO ADEQUADO E MAIS LARGO.

\begin{tabular}{|c|c|c|c|c|c|c|c|}
\hline $\begin{array}{l}\text { Idade } \\
\text { (meses) }\end{array}$ & & $\bar{d}$ & sd & $\mathbf{n}$ & $\mathbf{t}$ & t crítico & $\begin{array}{c}\text { signi- } \\
\text { cância } \\
1 \%\end{array}$ \\
\hline $\begin{array}{l}12 \vdash 24 \\
24 \vdash 36 \\
36 \vdash 48 \\
48 \vdash 60 \\
60 \vdash 72 \\
72 \vdash 84\end{array}$ & 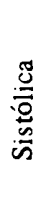 & $\begin{array}{l}9,8 \\
9,1 \\
9,3 \\
7,5 \\
8,0 \\
7,7\end{array}$ & $\begin{array}{l}7,66 \\
5,63 \\
6,82 \\
6,17 \\
6,60 \\
6,79\end{array}$ & $\begin{array}{l}43 \\
47 \\
60 \\
50 \\
51 \\
55\end{array}$ & $\begin{array}{r}8,39 \\
11,09 \\
10,56 \\
8,68 \\
8,57 \\
8,41\end{array}$ & $\begin{array}{l}2,704 \\
2,704 \\
2,704 \\
2,704 \\
2,704 \\
2,704\end{array}$ & $\begin{array}{l}\text { sim } \\
\text { sim } \\
\text { sim } \\
\text { sim } \\
\text { sim } \\
\text { sim }\end{array}$ \\
\hline $\begin{array}{l}12 \vdash 24 \\
24 \vdash 36 \\
36 \vdash 48 \\
48 \vdash 60 \\
60 \vdash 72 \\
72 \vdash 84\end{array}$ & 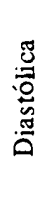 & $\begin{array}{l}8,2 \\
8,0 \\
6,4 \\
5,4 \\
5,2 \\
5,0\end{array}$ & $\begin{array}{l}5,80 \\
6,60 \\
7,11 \\
5,61 \\
6,40 \\
5,87\end{array}$ & $\begin{array}{l}43 \\
47 \\
60 \\
50 \\
51 \\
55\end{array}$ & $\begin{array}{l}9,32 \\
8,31 \\
7,00 \\
6,90 \\
5,80 \\
6,32\end{array}$ & $\begin{array}{l}2,704 \\
2,704 \\
2,704 \\
2,704 \\
2,704 \\
2,704\end{array}$ & $\begin{array}{l}\operatorname{sim} \\
\operatorname{sim} \\
\operatorname{sim} \\
\operatorname{sim} \\
\operatorname{sim} \\
\operatorname{sim}\end{array}$ \\
\hline
\end{tabular}


do, com bolsa de borracha de largura igual ao diâmetro do braço da criança, com acréscimo aproximado de $20 \%$, apresentaram oscilações em cada faixa etária, porém, estas diferenças não são significativas.

- Os valores médios encontrados para a PA em crianças de 1 a 6 anos de idade apresentaram a seguinte amplitude de variação: para a pressão sistólica de meninos entre $107,2(7,6) \mathrm{mmHg}-110,4(8,7) \mathrm{mmHg}$ e de $104,8(9,0) \mathrm{mmHg}$ a $107,9(6,4) \mathrm{mmHg}$ para a de meninas; e para a pressão diastólica, entre $75,3(6,9) \mathrm{mmHg}$ e $79,5(7,2)$ para meninos e de $75,3(5,9) \mathrm{mmHg}$ a $78,1(6,6) \mathrm{mmHg}$ para meninas. Estas diferenças entre os valores da PA de meninos e meninas não são significativas.

- Os valores das pressð̃es sistólica e diastólica dessas crianças sofreram influência da largura do manguito assim, quando se utilizou manguito mais estreito do que o adequado, os valores da PA se elevaram, tanto para a pressão sistólica como para a diastolica e, quando se utilizou manguito mais largo do que o adequado, os valores diminuíram tanto para a pressão sistólica como para a diastólica.

A elevação da PA observada ao utilizar manguito mais estreito do que o adequado teve a seguinte amplitude media de variação:

de 16,1 a $11,1 \mathrm{mmHg}$ para a pressão sistólica de meninos, e de 28,2 a $15,1 \mathrm{mmHg}$ para a pressão sistólica de meninas; de 11,9 a $9,3 \mathrm{mmHg}$ para a pressão diastólica de meninos, e de 18,8 a $6,7 \mathrm{mmHg}$ para a pressão diastólica de meninas.

A diminuição dos valores da PA observada ao utilizar manguito mais largo do que o adequado teve a seguinte amplitude média de variação:

de 10,2 a $7,0 \mathrm{mmHg}$ para a pressão sistólica de meninos, e de 9,8 a $7,5 \mathrm{mmHg}$ para a pressão sistólica de meninas; de 8,9 a $2,8 \mathrm{mmHg}$ para a pressão diastólica de meninos, e de 8,2 a $5,0 \mathrm{mmHg}$ para a pressão diastólica de meninas.

- De acordo com os resultados obtidos e adotando-se o critério de que a largura do manguito deva corresponder, aproximadamente, ao diâmetro do braço mais $20 \%$ para se verificar a PA de crianças, na prática diária seria necessário dispor-se de diferentes manguitos, cuja largura variasse pelo menos, de 1 em 1 centímetro.

- Para se obterem dados confiáveis da PA em crianças, toma-se necessário adequar a técnica da medida indireta à dimensão de seu braço, pela utilização de manguito cuja largura seja proporcional à circunferência do braço da criança e $20 \%$ maior que seu diâmetro. 
MARTINS, D.M.R. Arterial blood pressure estimative in children from one to six years old with special referente to the width of the cuff. . Rev. Esc. Enf. USP, São Paulo, 22 (3): 353-370, dec. 1988.

The author presents means and dispersion's mesures - standards deviations and percentiles - of systolic and diastolic pressures in children from one to six years old. The use of a cuff whose width equals the diameter of the child's arm plus an $20 \%$ increase was established. The study evidences a significant influence of this width upon the arterial blood pressure readings. So, when a narrower cuff was emplovedn, the average values of the systolic and diastolic pressures were overestimated and when a cuff large than the established one was used, they were underestimated.

UNITERMS: Blood pressure. Children.

\section{REFERẾNCIAS BIBLIOGRĀFICAS}

1. AlLEN-WilliamS, G.M. Pulse-rate and blood-pressure in infancy and early childhood. Arch. Dis. Child., London, 20: 125-8, 1945.

2. ASWORTH, A.M. et al.. Sphygmomanometer for the newborn. Lancet., London, 1:801-7, Apr. 1959.

3. BARKER, F.H. et al.. Standard method for taking and recording and recording blood pressure readings. J. Amer. Med. Ass., Chicago 113 (4): 294-7, July, 1939.

4. BERGEN, E.H. von et al.. Comparison of indirect and direct methods of manguina arterial blood pressure. J. Am. Heart Ass., 10 (4):481-90. Oct., 1954.

5. BERQUO, E.S. et al.. Bicestatística. São Paulo, E.P.U. 1981.

6. BORDLEY, J. et al.: Recommendations for human blood pressure determinations by sphygmomanometers. J. Am. Med. Ass., Chicago 147 (7):632-6, Oct., 1951.

7. BLUMENTHAL, S. et al.. Report of the force on blood pressure control in children. Pediatrics, tevanston, 59 (5): supl. 15-20, 1977.

8. BRITTON, S.V. Blood pressure measurement and hypertension in children. Pediat. Nurs., Pitman, 7 (4): 13-7, July/Aug. 1982.

9. DENES, F.T. et al.. Hipertensão arterial na infância - J. Ped., St . Louis, 42 (6): 36-44, gul. 1977.

10. FIORI, R.N. et al.. Prática pediátrica de urgência. São Paulo;'Artes Médicas, 1975, 350 p.

11. FUJIMURA, N.D. et al.. Hipertensão arterial. In: ALCANTARA, P. \& MARCONDES, E. Pediatria Básica. H. ed. São Paulo, Sarvier, 1947, p. 256.

12. GRAHAM, A.W. et al.. Blood pressure in children between the ages of five and sixteen years. Amer. J. Dis. Child., Chicago, 69 (4): 283-7, Apr., 1945.

13. GREENFIELD, D. et al.. Children can have high blood pressure, too. Amer. J. Nurs., New York, 75 (5): $770-2$, May 1976.

14. GONTEROTH, W.G. \& NADAS, A.S. Blood pressure measurement in infants and children. Pediat. Qin. N. Amer., Philadelphia, 2:257-63, Feb., 1955.

15. HAGGERTY, T.J. et al.. Essential hypertension in infancy and childhood. Amer. J. Dis. Child., Chicago, 92:535-49, 1956.

16. JELLIFFE, D.E. Avaluación del estado de nutrición de la comunidad. Genebra., Organizacion Mondial de la Sante, 1968.

17. KARVONEY, M.J. et al.. Sphygmomanometer cuff size and the accurasy of indirect measurement of blood pressure. Amer. J. Cardiol., New York, 18:688-98, May, 1964.

18. KIRKENDALL, W.M. et al.. Recomendations for human blood pressure determination b: sphygmomanometers. New York, American Heart Association, 1967,23 p. 
19. LANCOUR, J. How to avoid pitfalls in measuring blood pressure. Amer. J. Nurs., New York, 76 (5): 773-5, May, 1976.

20. MARCONDES, E. et al.. Estudo antropométrico de crianças brasileiras de zero a doze anos de idade. An. Nestlè., São Paulo, (84): 3-200, s.d.

21. MCLAREN, D.S. et al.. Urinary creatinine and hydroxiproline in relation to childhood malnutrition. Bril. J. Nutr., London, 24:614-51, 1970.

22. MARTINS, D.M.R. Verificação da pressão arterial em crianças: condiçōes e procedimentos. Rev. Esc. e Enf. USP., São Paula, 14 (2):179-83, ago. 1980.

23. MARTINS, D.M.R. Estudo da pressáo arterial no primeiro ano de vida. São Paulọ, 1978, 85 p. (Disser tação de mestrado - Escola de Enfermagem da USP).

24. MOSS, A.J. \& ADAMS, F.H. Auscultatory and intra-arterial pressure: a comparison in children, with special reference to cuff width. Brief. Clin. Lab. Observ., 66 (6): 1094-7, Jun. 1965.

25. MOSS, A.J. et al.. Measurement of arterial blood pressur. In:

Heart discase in infants children and adolescents. Baltimore, Williams \& Wilkins, 1968, cap. 5 p. 58-66.

26. MURAHOVSCHI, J. et al.. Emergências cardiocirculatórias. In: em pediatria Gomes de Mattos. 3. ed. São Paulo, Sarvier, 1976, cap. 6, p. 16790.

27. ROBINOW, M. et al.. Accuracy of clinical determinations of blood pressure in children: with values under normal and abnormal conditions. Amer. J. Dis. Child., Chicago, 58 (1):102-18, July, 1989.

28. SPIEGELL, M.R. Estatística. São Paulo, McGraw-Hill do Brasil, 1972.

29. WOODBURY, R.A. et al.. Blood pressure studies on infants. Amer. J. Physiol., Bcthesda, 122 (2): $472-9,1988$. 
ANEXO 1

INDICE DE MCLAREN

VALORES PARA O CÁLCULO DO IŃDICE DE MCLAREN.(1970).

PARA AS MEDIDAS DO PERIMETRO BRAQUIAL E PESO.

AMPLITUDES (em \%)

$$
\begin{gathered}
100 \text { e mais } \\
100-90 \\
90-80 \\
80-70 \\
70-60 \\
60 \text { e menos }
\end{gathered}
$$

GRAUS

0

1

2

3

4

5

PARA AS MEDIDAS DE ALTURA E PERIMETRO CEFÁliCO.

AMPLITUDES (em \%)

$$
\begin{gathered}
100 \text { e mais } \\
100-90 \\
90-85 \\
85-80 \\
80 \text { e menos }
\end{gathered}
$$

\section{GRAUS}

0
1
2
3
4

\section{CLASSIFICAÇÃO:}

Zero, 1 e 2 - crescimento normal.

$3,4,5,6,7$ e 8 - crescimento abaixo do normal.

9 e mais - crescimento muito prejudicado.

\section{OBSERVAÇÃO:}

A classificação aditada no presente estudo foi a seguinte: a soma dos pontos de 0,1 e 2 foi considerada crescimento normal; 3,4 e 5 crescimento levemente prejudicado; 6,7 e 8 crescimento moderadamente prejudicado e 9 e mais, crescimento muito prejudicado. 
FICHA PARA REGISTRO DE DADOS

DATA

IDENTIFICAÇÃO

\begin{tabular}{|c|c|c|c|}
\hline \multicolumn{2}{|l|}{ NOME } & \multicolumn{2}{|c|}{ LOCAL } \\
\hline \multicolumn{4}{|c|}{ DATA DE NASCIMENTO ______ IDADE_ } \\
\hline \multicolumn{4}{|c|}{ INDICE DE CRESCIMENTO } \\
\hline MEDIDAS & $\mathrm{cm}$ ou $\mathrm{Kg}$ & $\%$ & GRAU \\
\hline $\begin{array}{l}\text { Peso } \\
\text { Altura } \\
\text { Perímetro cefálico } \\
\text { Perímetro braquial }\end{array}$ & & & $\begin{array}{l}\text { Indice de McLaren: } \\
\text { grau :_ } \%\end{array}$ \\
\hline
\end{tabular}

CONDIÇOES DA CRIANÇA

Aparência geral

Temperatura

Frequiência card íaca

Posição

Tempo de repouso

Freqüência respiratória

Largura do manguito

Estado emocional e relaxamento muscular

Observaçð̃es:

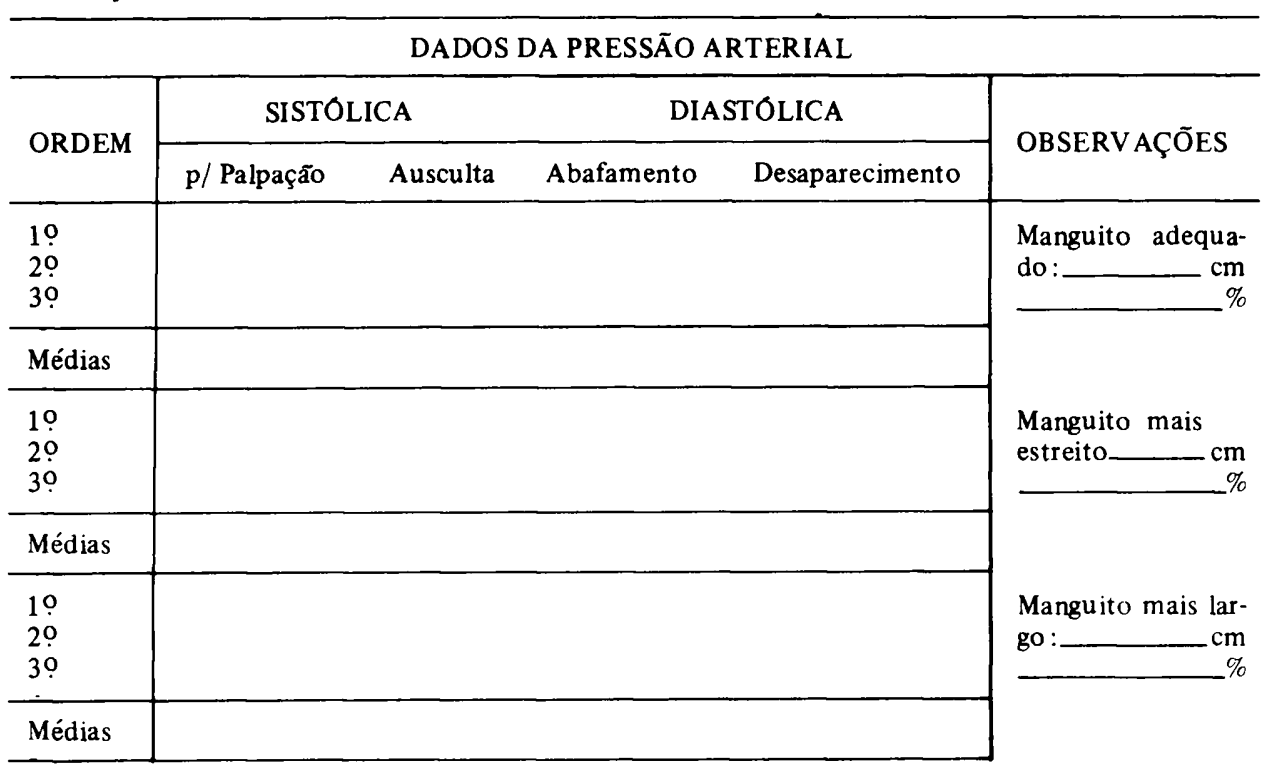




\section{ANEXO 3}

RELAÇÃO DE PERIMETROS BRAQUIAIS E CORRESPONDENTE LARGURA DE MANGUITOS, SEGUNDO O CRITÉRIO DO ACRESCIMO MÉDIO DE 20\% SOBRE O DIÂMETRO DO BRAÇO.

\begin{tabular}{cc}
$\begin{array}{c}\text { PERIMETRO BRAQUIAL } \\
(\mathrm{cm})\end{array}$ & $\begin{array}{c}\text { LARGURA DO MANGUITO } \\
(\mathrm{cm})\end{array}$ \\
\hline $9,5-10,1$ & 3,5 \\
$10,1-11,2$ & 4,0 \\
$11,2-12,5$ & 4,5 \\
$12,5-13,8$ & 5,0 \\
$13,8-15,1$ & 5,5 \\
$15,1-16,4$ & 6,0 \\
$16,4-17,5$ & 6,5 \\
$17,5-18,9$ & 7,0 \\
$18,9-20,1$ & 7,5 \\
$20,1-21,1$ & 8,0 \\
$21,1-22,5$ & 8,5 \\
$22,5-24,0$ & 9,0 \\
\hline
\end{tabular}

Recebido para publicação em 11/08/87. 